\title{
Full-band polar optical phonon scattering analysis and negative differential conductivity in wurtzite GaN
}

\author{
C. Bulutay \\ Department of Physics, Bilkent University, 06533 Bilkent, Ankara, Turkey \\ B. K. Ridley \\ Department of Electronic Systems Engineering, University of Essex, Colchester C04 3SQ, United Kingdom \\ N. A. Zakhleniuk \\ Caswell Technology, Marconi Caswell, Towcester, Northants, NN12 8EQ, United Kingdom
}

(Received 27 March 2000; revised manuscript received 5 July 2000)

\begin{abstract}
GaN has promising features for high-field electronics applications. To scrutinize these transport-related properties, primarily the dominant scattering mechanism in this material needs to be well characterized. In the quest for Bloch oscillations in bulk GaN, our aim is to conduct a full-band scattering analysis requiring very high energies where parabolic approximation is far from applicable. For this purpose, we first obtain an accurate band structure for the conduction band of wurtzite GaN based on the empirical pseudopotential method, using the most recent experimental data as the input. We compute the scattering rate, relevant up to room temperatures, due to longitudinal-optical-like and transverse-optical-like polar phonon modes along several (high-symmetry) directions, from the conduction band minimum at the zone center to the half of the reciprocal lattice vector in each direction. We observe that the location and the symmetry of the neighboring valleys to the route play a decisive role on the scattering rates. The observation of Bloch oscillations in bulk wurtzite $\mathrm{GaN}$ is doomed by the very large value of the polar scattering rate. However, there exists the possibility of a negative differential conductivity driven by the negative effective mass part of the band structure for fields above $2.3 \mathrm{MV} / \mathrm{cm}$ for wurtzite $\mathrm{GaN}$.
\end{abstract}

\section{INTRODUCTION}

In the last decade GaN and other related III nitrides have been a subject of intensive research, initiated by their outstanding optoelectronic prospects, such as light emitting diodes, and lasers in the visible spectrum. ${ }^{1}$ The fact that these III nitrides are wide bandgap materials, also make them very attractive for electronic applications, especially for highpower and high-temperature purposes. The ability of these III nitrides to withstand very high electric fields on the order of few MV/cm, brings new possibilities, not within reach of the traditional semiconductors such as $\mathrm{Si}$ and GaAs. One intriguing possibility is to observe the elusive Wannier-Stark resonances and the associated Bloch oscillations in a bulk semiconductor, such as GaN., ${ }^{2,3}$ Such a study requires a detailed knowledge of the transport properties in these wide bandgap materials, primarily the characterization of the dominant scattering mechanism. This, in GaN is the polar optical phonon scattering due to its high degree of ionicity ${ }^{4}$ and hence large electron-phonon coupling constant.

The aim of this paper is to quantitatively analyze the full conduction band electron scattering rates due to polar optical phonon (POP) emission. We obtain these scattering rates in several different directions in the reciprocal lattice, from the conduction band minimum to the half of the reciprocal lattice vector in that direction. This comparative study also produces a valuable output for the likelihood of the WannierStark resonances in bulk wurtzite (WZ) GaN. For the accuracy of our results we base our analysis on realistic models for both electrons and phonons in $\mathrm{WZ} \mathrm{GaN}$.
The full-band scattering rate requires a reliable band energy and wave function knowledge. Empirical pseudopotential method $(E P M)^{5}$ is widely used for such purposes and several results for GaN have already been published. ${ }^{6-10} \mathrm{We}$ have analyzed these proposed band structures and found them to be unsatisfactory for the conduction band properties, such as the conduction band effective mass and also in the agreement to the more recently released experimental data on the conduction band energies. ${ }^{11,12}$ As this point is of central importance in our work, we have also performed an EPM study for WZ GaN, demanding a close fit to these conduction band properties. Based on this band structure, as a byproduct we present the energy levels and effective mass parameters of the conduction band satellite valleys, which play important role in high-field electronic transport properties.

Formally, the electron-phonon coupling in $\mathrm{WZ} \mathrm{GaN}$ is different from the well-known cubic case, bringing in longitudinal optical (LO)-like and transverse optical (TO)-like modes, ${ }^{13}$ rather than a single LO mode as in a zinc blende crystal having an isotropic phonon spectrum. We analyze both of these modes and observe the TO-like scattering rate to be more than two orders of magnitude lower than the LO-like modes. Furthermore, we find the LO scattering rate in the cubic approximation to be in very good agreement with the LO-like case, regardless of the chosen point in the Brillouin zone (BZ).

The symmetry of the initial- and final-state electron wave functions plays an important role in the correct assessment of the scattering rates. Indeed, we observe that above the energy of the lowest satellite valley $U_{3, \min }$ the rates are significantly 
overestimated if the cell-periodic overlap is taken as unity, which actually works well only in the vicinity of the conduction band minimum. Based on these directional scattering rates we investigate the high-field negative differential conductivity possibilities.

In the next section, we present our guidelines and results for the band structure fitting, concentrating on the conduction band properties. In Sec. III the formulation for the POP scattering for the WZ crystal is given. Our results are contained in Sec. IV, followed by a discussion in Sec. V on the implications of these results in connection to Bloch oscillations and negative differential conductivity. A brief summary is given in Sec. VI. The Appendix discusses two different implementations of computing the scattering rate over the BZ.

\section{BAND STRUCTURE FITTING FOR THE CONDUCTION BAND}

EPM admirably suits to our purpose of computing the scattering rate, as it provides with reasonable effort an empirically tailored band profile at the same time yielding the pseudowave functions necessary for the rigorous treatment of the transition matrix elements. Several groups have already obtained the band structure of $\mathrm{WZ}$ GaN using EPM ${ }^{6-10}$ reporting their form factors as well, which enables other researchers to circumvent the task of fitting to the experimental values. Apparently, the emphasis in these fitting procedures has been on the valence band (VB) structure, predominantly due to its importance in optoelectronic oriented applications. As for the conduction band (CB), the fitted EPM band structure reported by these groups ${ }^{7-10}$ have important drawbacks; notably, the CB minimum effective mass comes out to be $0.15 m_{0}$ or less, whereas the experimentally measured ${ }^{14}$ value for WZ GaN (after subtracting the polaronic contribution) is $0.2 m_{0}$, with $m_{0}$ being the free electron mass. This discrepancy is quite significant for the low-field electronic transport properties and has even more drastic consequences for higher energies. Furthermore, there have been two very recent experimental reports regarding the band structure of the $\mathrm{CB}$ which were not available at the time the previous EPM works were carried out. First, Valla et al. have measured the $\mathrm{CB}$ structure of $\mathrm{WZ} \mathrm{GaN}$ along the $\Gamma-\mathrm{A}$ direction in the BZ using the inverse photoemission techniques. ${ }^{11}$ Secondly, Sun et al. ${ }^{12}$ analyzing the ultrafast electron dynamics in $n$-doped $\mathrm{GaN}$ have reported the energy separation between the lowest satellite valley $U_{3, \min }$ along the $U$ symmetry line and the CB minimum (as $1.34 \mathrm{eV}$ ). In the lack of any further verification of these results, we comply with these experiments and use them in our fitting procedure. Table I lists the chosen target values and the corresponding attained values by the optimization which was based on the Metropolis algorithm. ${ }^{17}$

The fact that we have favored the CB properties, leads to some discrepancy in the VB structure and more significantly the bandgap value comes out $1 \mathrm{eV}$ higher. This stems from the requirement of a heavier $\mathrm{CB}$ effective mass to meet the experimental value that results in pushing the $\mathrm{VB}$ and $\mathrm{CB}$ apart. As a matter of fact, Kolník et al. have also encountered this problem in their EPM fitting to the $\mathrm{CB}$, which they remedied by rigidly shifting the $\mathrm{CB}$, so that the true experi-
TABLE I. Target values based on the quoted references and the attained values by our optimization. All energies are given in $\mathrm{eV}$. The lineup from top to bottom crudely represents the emphasis we used in the optimization of the parameters, the last items being poorly included to the overall cost function. For the bandgap value $E_{g}$ we do not use the attained value $(4.52 \mathrm{eV})$, but rather, the target value $(3.5 \mathrm{eV})$ by rigidly shifting all $\mathrm{CB}$ with respect to $\mathrm{VB}$.

\begin{tabular}{ccc}
\hline \hline & Target & Attained \\
\hline$m_{c \perp}^{*} / m_{0}, m_{c z}^{*} / m_{0}$ & $0.2,0.2$ (Ref. 14) & $0.200,0.196$ \\
$\Gamma_{3}^{c}-\Gamma_{1}^{c}$ & 2.13 (Ref. 11) & 2.14 \\
$A_{1,3}^{c}-\Gamma_{1}^{c}$ & 3.0 (Ref. 11) & 2.52 \\
$U_{3, \text { min }}^{c}-\Gamma_{1}^{c}$ & 1.34 (Ref. 12) & 1.34 \\
$E_{g}$ & 3.50 (Ref. 16) & $4.52 \rightarrow 3.50$ \\
$\Gamma_{6}^{v}-\Gamma_{3}^{v}$ & 6.0 (Ref. 15) & 6.0 \\
$\Gamma_{6}^{v}-\Gamma_{5}^{v}$ & 1.0 (Ref. 15) & 0.74 \\
$\Gamma_{6}^{v}-\Gamma_{1}^{v}$ & 0.015 (Ref. 16) & -0.025 \\
\hline \hline
\end{tabular}

mental band gap of $3.5 \mathrm{eV}$ was restored. ${ }^{18} \mathrm{We}$ apply the same correction here. Figure 1 displays our resultant band structure; for comparison, the lowest two $\mathrm{CB}$ of the band structure due to Yeo et al. $^{8}$ are also shown. There is a significant disagreement between the two, which we believe to be in favor of our results, based on our emphasis on the CB and fitting to more recent experimental results. ${ }^{11,12}$ Using this band structure, in Table II we report the energy levels of the several satellite valleys in the CB as well as their effective mass parameters. These values are yet to confront experiment, however, qualitatively it can be noted that compared to the $\mathrm{CB}$ minimum $\left(\Gamma_{1}\right.$ valley), the effective masses are heavier in all these satellites and anisotropic, futhermore the $\mathrm{L}$ point turns out to be a saddle point rather than a valley, therefore diminishing its significance in scattering processes. In Fig. 2 we show the associated density of states (DOS) per spin, which directly comes into play in the deformation potential phonon scattering. The sudden increase in CB DOS at $4.84 \mathrm{eV}$ is due to the $U_{3, \min }$ satellite valley, signaling its importance in the electronic transport properties.

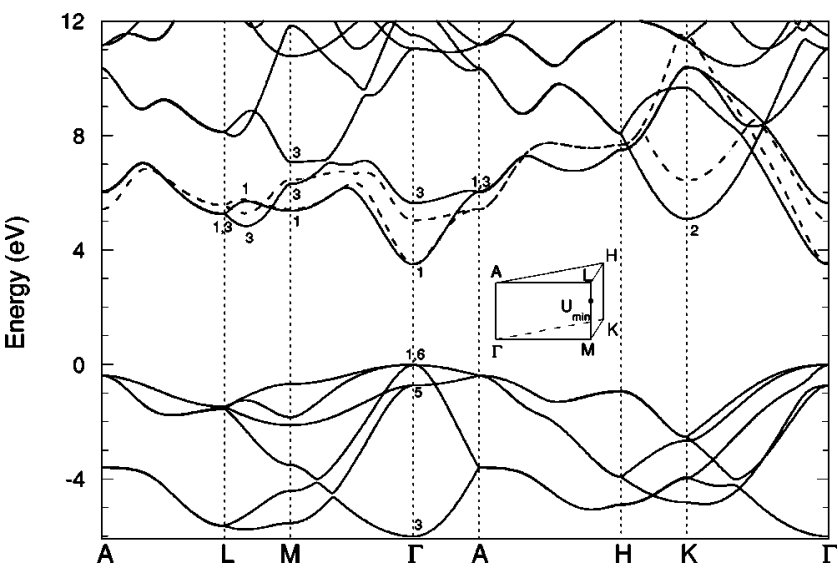

FIG. 1. Energy band structure for $\mathrm{WZ}$ GaN based on EPM, fitted to recent experimental $\mathrm{CB}$ data. For comparison purposes, the lowest two CB reproduced from the form factors of Yeo et al. (Ref. 8) are also included (dashed lines). Several important points are labeled and the inset shows the irreducible part of the BZ (not drawn to scale). 
TABLE II. The energy and the effective mass parameters of the $\mathrm{CB}$ minimum and the other satellite valleys. The energy reference is taken as that of the $\mathrm{CB}$ minimum. $m_{\Gamma M}, m_{\Gamma K}$, and $m_{\Gamma A}$ represent the effective masses along the $\Gamma-M, \Gamma-K$, and $\Gamma-A$ directions, respectively.

\begin{tabular}{lcccc}
\hline \hline & $E_{X}(\mathrm{eV})$ & $m_{\Gamma M} / m_{0}$ & $m_{\Gamma K} / m_{0}$ & $m_{\Gamma A} / m_{0}$ \\
\hline$\Gamma_{1}$ & 0.00 & 0.200 & 0.200 & 0.196 \\
$U_{3, \text { min }}$ & 1.34 & 0.879 & 0.337 & 0.293 \\
$K_{2}$ & 1.59 & 0.547 & 0.547 & 0.347 \\
$L_{1,3}$ & 1.75 & 0.865 & 0.297 & -0.018 \\
$M_{1}$ & 1.87 & 0.887 & 0.189 & 1.078 \\
$\Gamma_{3}$ & 2.14 & 0.247 & 0.247 & 1.390 \\
\hline \hline
\end{tabular}

The key ingredients in EPM are the fitted pseudopotential form factors, for which we use the following forms:

$$
\begin{aligned}
& V_{s}(q)=\left(s_{1} q^{3}+s_{2} q^{2}+s_{3}\right) \exp \left(-s_{4} q^{s_{5}}\right), \\
& V_{a}(q)=\left(a_{1} q^{2}+a_{2} q+a_{3}\right) \exp \left(-a_{4} q^{a_{5}}\right),
\end{aligned}
$$

where $V_{s}$ and $V_{a}$ are the symmetric and antisymmetric form factors ${ }^{5}$ in Rydbergs, $q$ is the wave number in units of $2 \pi / a$ with $a$ being the lattice constant in the hexagonal plane, and $s_{i}, a_{i}(i=1, \ldots, 5)$ are the fitting parameters which are listed in Table III. We do not include the spin-orbit interaction, having small effect on the CB due to dominant $s$ symmetry of the corresponding wave functions. The other parameters of the EPM are also given in Table III.

\section{EXPRESSIONS FOR THE POP SCATTERING RATE IN A UNIAXIAL CRYSTAL}

Based on Fermi's golden rule, the one-phonon scattering rate of an electron at the band $m$, with a wave vector $\mathbf{k}$, having an energy $E_{m}(\mathbf{k})$, is given by

$$
\begin{aligned}
W_{m, j}^{ \pm}(\mathbf{k})= & \sum_{m^{\prime}, \mathbf{k}^{\prime}} \frac{2 \pi}{\hbar}\left|\left\langle m^{\prime}, \mathbf{k}^{\prime} ; n_{j, \mathbf{q}} \pm 1\left|H_{e-\mathrm{ph}}^{j}\right| m, \mathbf{k} ; n_{j, \mathbf{q}}\right\rangle\right|^{2} \\
& \times \delta\left[E_{m^{\prime}}\left(\mathbf{k}^{\prime}\right)-E_{m}(\mathbf{k}) \pm \hbar \omega_{j, \mathbf{q}}\right]
\end{aligned}
$$

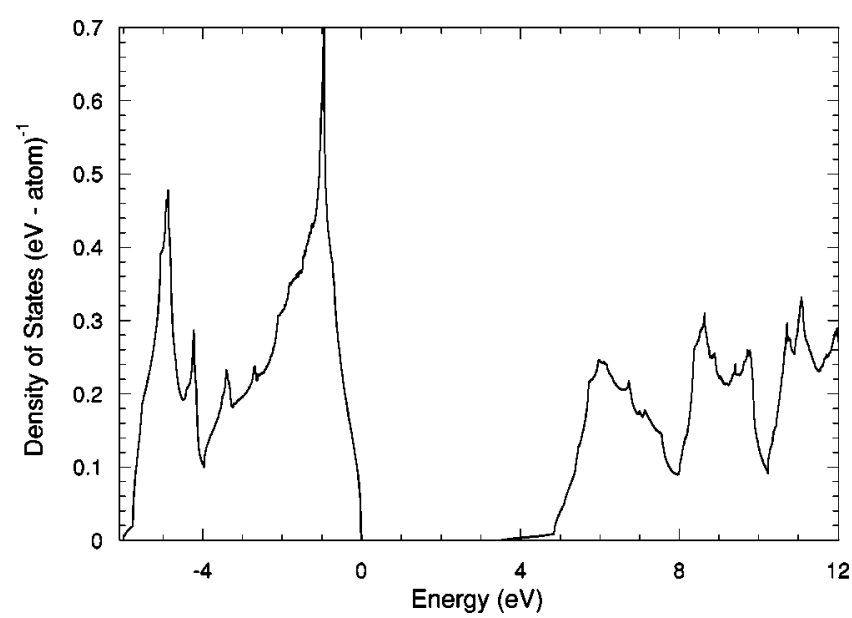

FIG. 2. Density of states per spin associated with the band structure shown in Fig. 1.
TABLE III. Fitted values for the constants in the pseudopotential form factors $V_{s}$ and $V_{a}$; see Eqs. (1) and (2). Other parameters used in the EPM are also listed, where $a$ and $c$ are the lattice constants in the hexagonal plane and along the $c$ axis, respectively, $u$ is the WZ internal structural parameter, and $E_{\max }$ denotes the radius of the energy sphere used for the reciprocal lattice vectors.

\begin{tabular}{lc}
\hline \hline$s_{1}=0.1575$ & $a_{1}=-0.1300$ \\
$s_{2}=0.0763$ & $a_{2}=0.4177$ \\
$s_{3}=-1.1286$ & $a_{3}=0.2638$ \\
$s_{4}=1.1471$ & $a_{4}=0.6206$ \\
$s_{5}=0.9462$ & $a_{5}=1.7105$ \\
\hline$a=3.1879 \AA$ & \\
$c=5.1851 \AA$ & \\
$u=0.3760$ & \\
$E_{\max }=15 \mathrm{Ry}$ & \\
\hline
\end{tabular}

where the primed indices represent the final-state electron labels over which a summation is performed, $\mathbf{q}$ is the phonon wave vector having an energy $\hbar \omega_{j, \mathbf{q}}$; the index $j$ labels the particular phonon branch taking part in scattering and $n_{j, \mathbf{q}}$ is the thermal population of this specific phonon. In the case of double signs, upper (lower) sign refers to phonon emission (absorption) process. $H_{e \text {-ph }}^{j}$ is the electron-phonon interaction operator, given by

$$
\begin{aligned}
& H_{e-\mathrm{ph}}^{j}=\frac{1}{V} \int_{V} d^{3} r \sum_{m_{2}, \mathbf{k}_{2}} e^{-i \mathbf{k}_{2} \cdot \mathbf{r}} u_{m_{2}, \mathbf{k}_{2}}^{*}(\mathbf{r}) b_{m_{2}, \mathbf{k}_{2}}^{\dagger} \sum_{\mathbf{q}} C_{j}(\mathbf{q}) \\
& \times\left[a_{j, \mathbf{q}} e^{i \mathbf{q} \cdot \mathbf{r}}-a_{j, \mathbf{q}}^{\dagger} e^{-i \mathbf{q} \cdot \mathbf{r}}\right] \sum_{m_{1}, \mathbf{k}_{1}} e^{i \mathbf{k}_{1} \cdot \mathbf{r}} u_{m_{1}, \mathbf{k}_{1}}(\mathbf{r}) b_{m_{1}, \mathbf{k}_{1}},
\end{aligned}
$$

here, $V$ is the total crystal volume, $C_{j}(\mathbf{q})$ is the electronphonon coupling coefficient, $b_{m, \mathbf{k}}$ and $a_{j, \mathbf{q}}$ are, respectively, the electron and phonon annihilation operators. $u_{m, \mathbf{k}}(\mathbf{r}) \mathrm{de}-$ notes the cell-periodic part of the electron wave function, which in the EPM has the expansion

$$
u_{m, \mathbf{k}}(\mathbf{r})=\sum_{l} a_{l}(m, \mathbf{k}) e^{i \mathbf{G}_{l} \cdot \mathbf{r}}
$$

where $\mathbf{G}_{l}$ 's are the reciprocal lattice vectors. Equation (4) in Eq. (3) yields

$$
\begin{aligned}
W_{m, j}^{ \pm}(\mathbf{k})= & \frac{2 \pi}{\hbar} \sum_{m^{\prime}, \mathbf{k}^{\prime}} \Delta_{m^{\prime}, m}\left(\mathbf{k}^{\prime}, \mathbf{k}\right)\left|C_{j}(\mathbf{q})\right|^{2}\left(n_{j, \mathbf{q}}+\frac{1}{2} \pm \frac{1}{2}\right) \\
& \times \delta\left[E_{m^{\prime}}\left(\mathbf{k}^{\prime}\right)-E_{m}(\mathbf{k}) \pm \hbar \omega_{j, \mathbf{q}}\right]
\end{aligned}
$$

with the constraint $\mathbf{q}=\mp\left(\mathbf{k}^{\prime}-\mathbf{k}\right)$ mapped to first BZ, which applies if we discard umklapp processes as they have minor role in POP scattering; note that in the rest of the paper all wave vectors lie in the first BZ, i.e., reduced wave vectors. In Eq. (6) $\Delta_{m^{\prime}, m}\left(\mathbf{k}^{\prime}, \mathbf{k}\right)$ is defined as

$$
\Delta_{m^{\prime}, m}\left(\mathbf{k}^{\prime}, \mathbf{k}\right)=\left|\frac{1}{\Omega} \int_{\Omega} u_{m^{\prime}, \mathbf{k}^{\prime}}^{*}(\mathbf{r}) u_{m, \mathbf{k}}(\mathbf{r}) d^{3} r\right|^{2},
$$


where the integration is over the primitive cell volume, $\Omega$. Using Eq. (5), the above integration turns into a summation over the reciprocal lattice vectors as

$$
\Delta_{m^{\prime}, m}\left(\mathbf{k}^{\prime}, \mathbf{k}\right)=\left|\sum_{l} a_{l}^{*}\left(m^{\prime}, \mathbf{k}^{\prime}\right) a_{l}(m, \mathbf{k})\right|^{2},
$$

with the $a_{l}$ 's readily computed by EPM.

As the WZ structure belongs to the class of uniaxial crystals, the electron-polar optical phonon coupling coefficient is formally different from the well-known cubic (Fröhlich) form. ${ }^{20}$ For the case of a weakly anisotropic uniaxial crystal, Lee and co-workers ${ }^{21}$ have obtained the following approximate forms for the coupling coefficients of the longitudinaloptical (LO)-like and transverse-optical (TO)-like modes

$$
\begin{aligned}
\left|C_{\mathbf{q}}^{\text {LO-like }}\right|^{2} \simeq & \frac{2 \pi e^{2} \hbar}{V q^{2} \Omega_{L}\left(\theta_{\mathbf{q}}\right)} \\
& \times\left[\frac{\sin ^{2} \theta_{\mathbf{q}}}{\left(\frac{1}{\epsilon_{\perp}^{\infty}}-\frac{1}{\epsilon_{\perp}^{0}}\right) \omega_{\perp L}^{2}}+\frac{\cos ^{2} \theta_{\mathbf{q}}}{\left(\frac{1}{\epsilon_{z}^{\infty}}-\frac{1}{\epsilon_{z}^{0}}\right) \omega_{z L}^{2}}\right]^{-1}
\end{aligned}
$$

$$
\begin{aligned}
& \left|C_{\mathbf{q}}^{\text {TO-like }}\right|^{2} \\
& \qquad=\frac{2 \pi e^{2} \hbar}{V q^{2} \Omega_{T}\left(\theta_{\mathbf{q}}\right)} \frac{\left(\omega_{\perp}^{2}-\omega_{z}^{2}\right)^{2} \sin ^{2} \theta_{\mathbf{q}} \cos ^{2} \theta_{\mathbf{q}}}{\left(\epsilon_{\perp}^{0}-\epsilon_{\perp}^{\infty}\right) \omega_{\perp}^{2} \cos ^{2} \theta_{\mathbf{q}}+\left(\epsilon_{z}^{0}-\epsilon_{z}^{\infty}\right) \omega_{z}^{2} \sin ^{2} \theta_{\mathbf{q}}},
\end{aligned}
$$

where $\theta_{\mathbf{q}}$ is the angle phonon wave vector, $\mathbf{q}$ makes with the crystal $c$ axis, $\omega_{\perp}\left(\omega_{z}\right)$ is the TO phonon frequency perpendicular to (along) the $c$ axis, $\omega_{\perp L}\left(\omega_{z L}\right)$ is the LO phonon frequency perpendicular to (along) the $c$ axis, $\epsilon_{\perp}^{\infty}\left(\epsilon_{z}^{\infty}\right)$ is the high frequency dielectric constant perpendicular to (along) the $c$ axis. The corresponding static dielectric constants are given by $\epsilon_{\perp}^{0}=\epsilon_{\perp}^{\infty} \omega_{\perp L}^{2} / \omega_{\perp}^{2}$ and $\epsilon_{z}^{0}=\epsilon_{z}^{\infty} \omega_{z L}^{2} / \omega_{z}^{2}$. The phonon frequency, $\omega_{j, \mathbf{q}}$, in Eq. (6) is now replaced for the LO-like and TO-like modes by $\Omega_{L}\left(\theta_{\mathbf{q}}\right)$ and $\Omega_{T}\left(\theta_{\mathbf{q}}\right)$, respectively, given by ${ }^{13}$

$$
\begin{gathered}
\Omega_{L}\left(\theta_{\mathbf{q}}\right)=\sqrt{\omega_{z L}^{2} \cos ^{2} \theta_{\mathbf{q}}+\omega_{\perp L}^{2} \sin ^{2} \theta_{\mathbf{q}}}, \\
\Omega_{T}\left(\theta_{\mathbf{q}}\right)=\sqrt{\omega_{z}^{2} \sin ^{2} \theta_{\mathbf{q}}+\omega_{\perp}^{2} \cos ^{2} \theta_{\mathbf{q}}}
\end{gathered}
$$

The nonzero TO-like coupling coefficient is due to the LO ingredient in this mode; its contribution vanishes for the isotropic case when $\omega_{\perp}=\omega_{z}$ and $\omega_{\perp L}=\omega_{z L}$, where the cubic form is restored.

The quantity, $W_{m}(\mathbf{k})$ gives the rate for an electron that belongs to band $m$, with a wave vector $\mathbf{k}$; for brevity, here we suppress some of the subscripts and superscripts. We can also introduce the following quantity that replaces the magnitude of $\mathbf{k}$ with the energy, while retaining the band index and the direction in the BZ, as

$$
\widetilde{W}_{m}^{\hat{\mathbf{k}}}(E)=\frac{\sum_{\mathbf{p}} W_{m}(\mathbf{p}) \delta(\hat{\mathbf{k}} \cdot \hat{\mathbf{p}}-1) \delta\left[E-E_{m}(\mathbf{p})\right]}{\sum_{\mathbf{p}} \delta(\hat{\mathbf{k}} \cdot \hat{\mathbf{p}}-1) \delta\left[E-E_{m}(\mathbf{p})\right]},
$$

where $\hat{\mathbf{p}}$ and $\hat{\mathbf{k}}$ represent unit vectors in the direction of $\mathbf{p}$ and $\mathbf{k}$, respectively. The denominator equals the number of crossings $^{22}$ of the value $E$ by the constant energy surface of the band $m$ along the vector $\hat{\mathbf{k}}$. The explicit energy dependence offered by $\widetilde{W}$ is sometimes more illuminating, and in our case it also enables direct comparison of the rates along different directions in the BZ.

\section{DIRECTIONAL POP SCATTERING RATES}

Based on the acceleration theorem, ${ }^{19}$ carriers in a band under an electric field $\mathbf{F}$ obey the relation $e \mathbf{F}=\hbar \mathbf{k}$. That is, carriers follow the trajectory along the field direction in the reciprocal space until a scattering event takes place, the dominant one being POP scattering in $\mathrm{GaN}$ due to its high ionicity. ${ }^{4}$ Note that, the scattering rate formulation in the previous section does not address an electric field. Indeed, Bardeen and Shockley ${ }^{23}$ have asserted that, acceleration of electrons by a uniform applied field, and scattering by lattice vibrations or imperfections are independent processes by showing that although the details of the transition may be altered by the electric field, the final expressions for the scattering rate are independent of the field. ${ }^{24,25}$ Accordingly, we trace the POP scattering rate of $\mathrm{CB}$ electrons along the highsymmetry directions, starting from $\mathrm{CB}$ minimum $\left(\Gamma_{1}\right)$ until half of the corresponding reciprocal lattice vector (RLV) along the lines $\Gamma-M, \Gamma-K$, and $\Gamma-A$, as well as along the $\Gamma-U_{3, \min }$ direction as this route connects the lowest two minima in the $\mathrm{CB}^{26}$ The trajectories along $\Gamma-K$ and $\Gamma-U_{3, \min }$ require longer RLVs and extend beyond the first BZ boundary. Another technical detail here is that EPM labels the bands by sorting their energies at each $\mathbf{k}$ point, regardless of the actual continuation of the bands beyond the band crossings. ${ }^{27}$ This point is particularly important for the band along the $\Gamma-K$ direction. For such crossing bands, we compare the values of the overlap parameter $\Delta_{m^{\prime}, m}$, given by Eqs. (7), (8) and determine which way the band actually continues. Further details about the implementation of the computation are given in the Appendix. A rewarding use of the overlap test is along the $\Gamma$ - $A$ direction, which reveals that there is actually a BZ folding at the $A$ point. This is due to four-layer stacking of the gallium and nitrogen atoms along the $c$ axis. ${ }^{29}$ So, an electron from the lower CB reaching the BZ boundary $(\mathbf{k}=\hat{\mathbf{z}} \pi / c)$ continues, in the reduced band scheme, to the upper band, starting from the point $-\hat{\mathbf{z}} \pi / c$. The Bloch oscillation cycle and the BZ folding effect are illustrated in Fig. 3. Hence, in tracing the scattering rate along this direction, we take this fact into account and follow the extended trajectory $\Gamma_{1}-A-\Gamma_{3}$, corresponding to segments $1-2$ in Fig. 3. The presence of the BZ folding modifies the POP scattering rates as the valley along the $\Gamma-A$ direction is now mapped back to $\Gamma$ point (i.e., $\Gamma_{3}$ valley), which somewhat increases the scattering rate to this valley from directions such as $\Gamma-M$, due to a smaller required pho- 


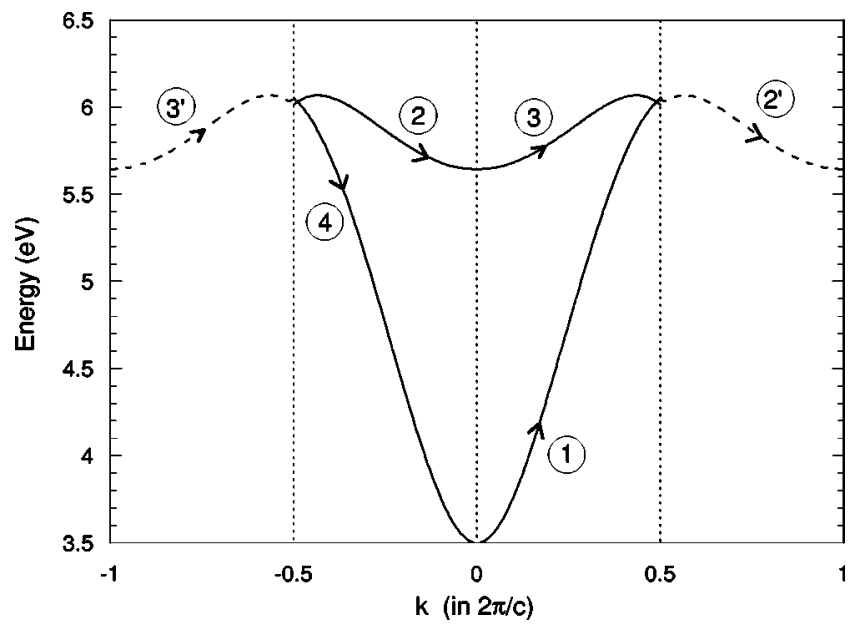

FIG. 3. Illustration of the BZ folding along the $\Gamma-A$ direction, showing the lowest "two" CB. The Bloch oscillation cycle following the sequence 1-2-3-4 in the folded BZ, corresponds to 1$2^{\prime}-3{ }^{\prime}-4$ in the unfolded case.

non wave vector compared to an unfolded-BZ case, enhancing the electron-phonon coupling [see Eqs. (9), (10)].

We use the following data for the GaN phonons: ${ }^{28,21}$ $\hbar \omega_{z}=66.08 \mathrm{meV}, \hbar \omega_{z L}=91.13 \mathrm{meV}, \hbar \omega_{\perp}=69.55 \mathrm{meV}$,
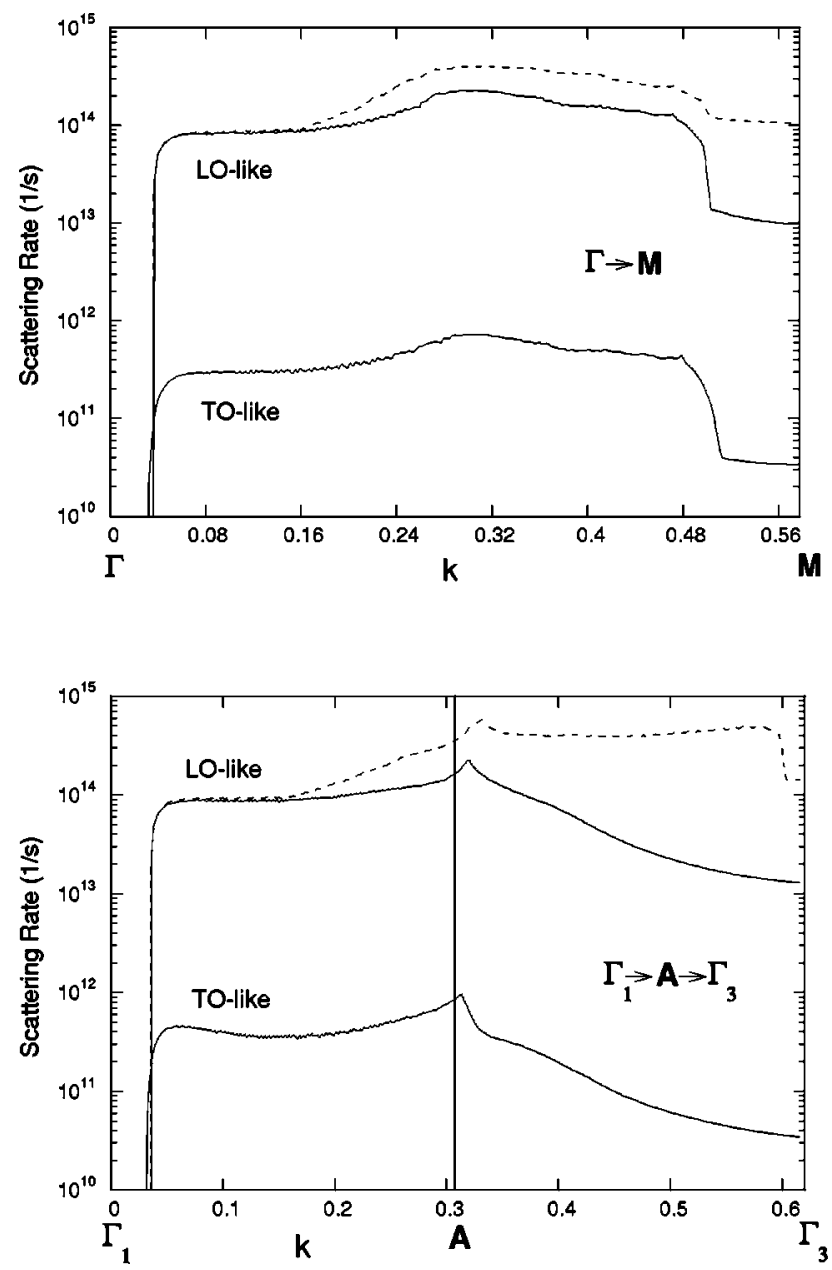

$\hbar \omega_{\perp L}=92.12 \mathrm{meV}$. As the LO-like optical phonon energies are more than 3.5 times the thermal energy at room temperature $(\sim 25 \mathrm{meV})$, the corresponding phonon occupation probability, $n_{j, \mathbf{q}}$ becomes negligible up to room temperatures; by the same token, POP absorption processes will be quite insignificant. So, we can essentially set $n_{j, \mathbf{q}} \equiv 0$ in Eq. (6) and drop the temperature parameter from our analysis and focus on POP emission only. Also, we use $\epsilon_{\perp}^{\infty}=\epsilon_{z}^{\infty}$ $=5.29$; these high-frequency dielectric permittivities are due to electronic polarization and for $\mathrm{GaN}$ they are usually assumed to be isotropic, ${ }^{28,21}$ unlike the anisotropic static counterparts having the ionic contributions as well. Figure 4 displays the LO-like and TO-like POP scattering rates along the chosen directions. The TO-like polar scattering branch is a hallmark of the WZ structure, but was not studied thoroughly over the full BZ. Here, we observe for WZ GaN that the TO-like rate is more than two orders of magnitude weaker than the LO-like mode over the whole extend of the BZ and for all four directions considered. It was recently suggested within low-energy effective mass approximation that the $\mathrm{cu}$ bic POP coupling constant is highly sufficient for the WZ $\mathrm{GaN} .{ }^{21}$ We have verified this by setting $\omega_{z} \rightarrow \omega_{\perp}$ and $\omega_{z L}$ $\rightarrow \omega_{\perp L}$ and the deviation from the LO-like results (not shown) was found to be typically less than $5 \%$ throughout
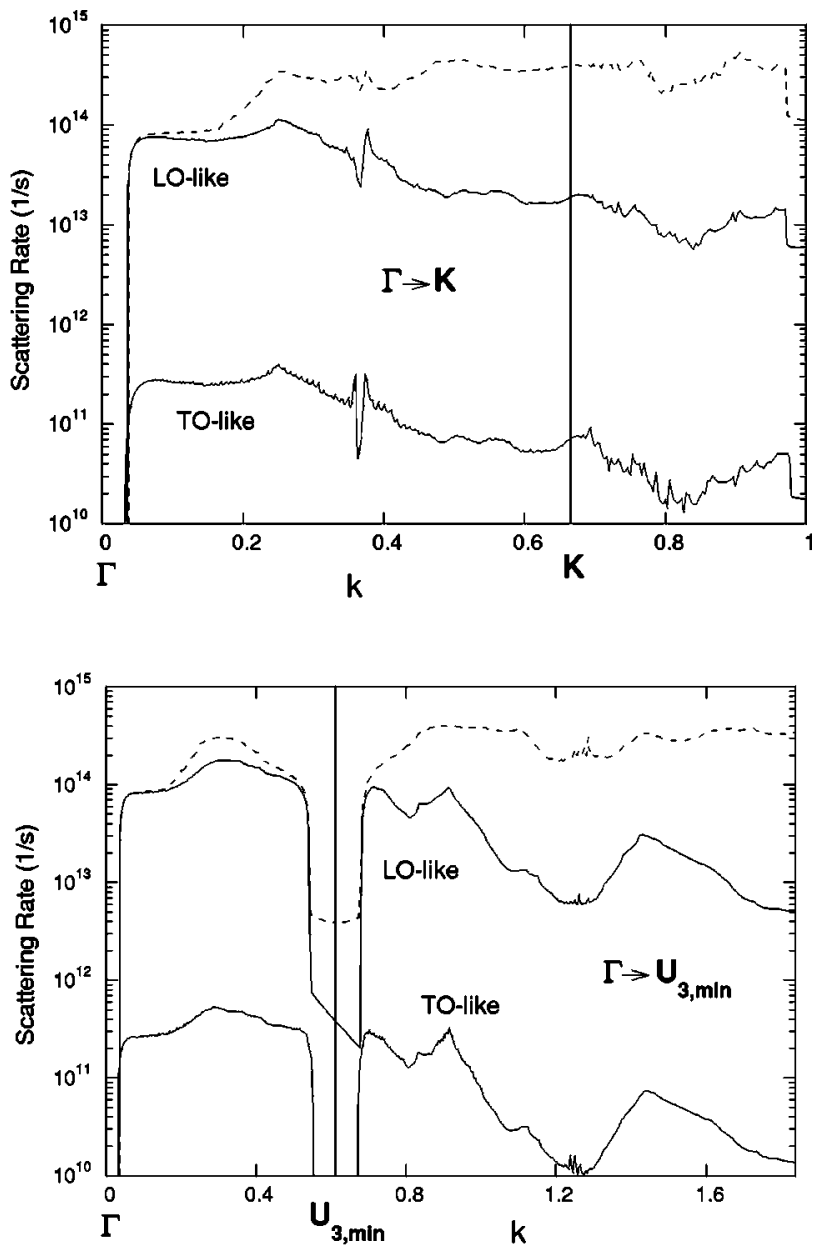

FIG. 4. Scattering rate versus wave vector (in units of $2 \pi / a$ ) along the directions $\Gamma-M, \Gamma-K, \Gamma_{1}-A-\Gamma_{3}, \Gamma-U_{3, \min }$ computed till half of the corresponding RLV; results apply up to room temperature. The dashed lines indicate the LO-like rates when the cell-periodic overlaps are taken to be unity. 


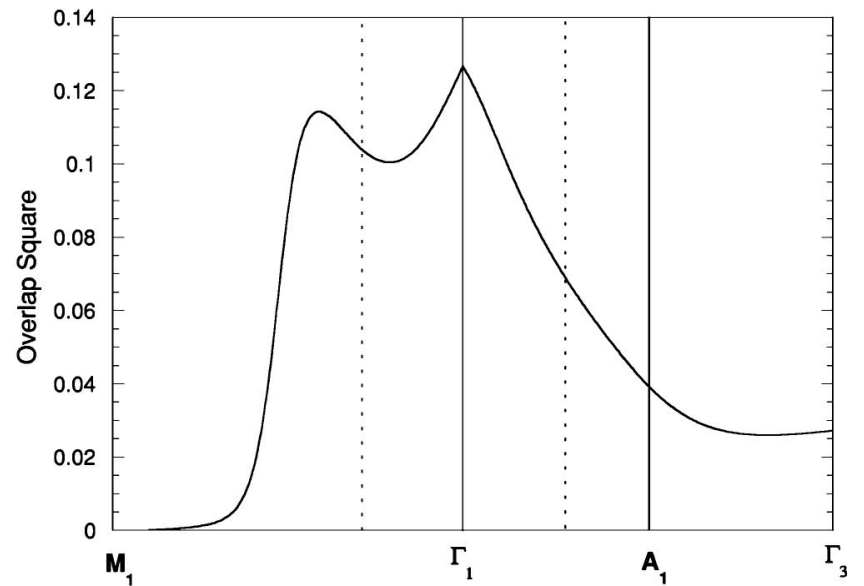

(a)

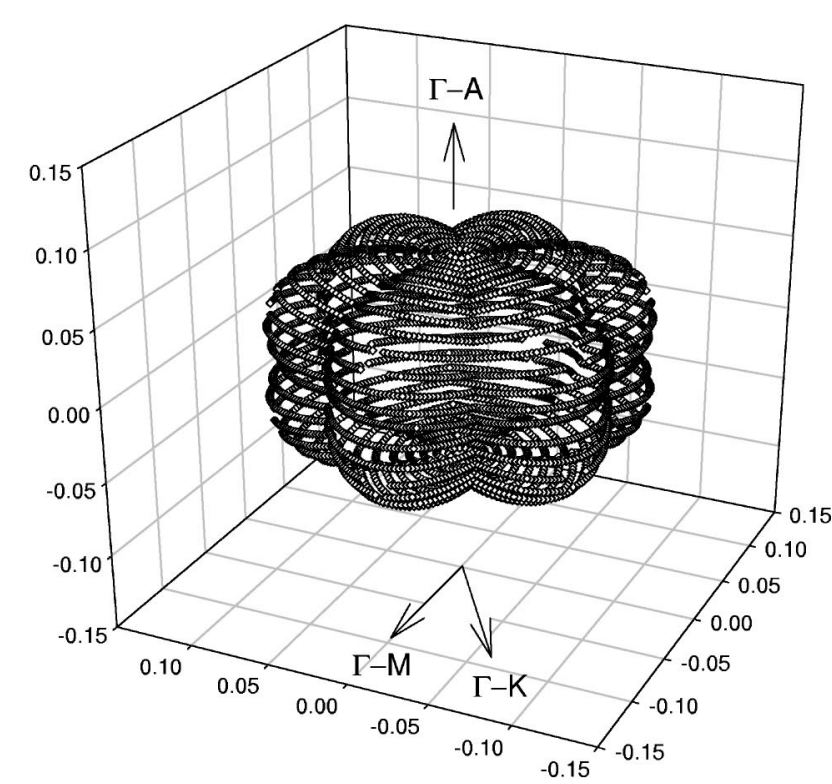

(b)

FIG. 5. The overlap parameter $\Delta_{m^{\prime}, m}$, variation with the finalstate point fixed at the $U_{3, \min }$ point. (a) The horizontal axis represents the initial-state points belonging to lowest $\mathrm{CB}$ lying along the $\Gamma-M$ and $\Gamma_{1}-A-\Gamma_{3}$ directions. The interval between the dashed lines corresponds to energies where POP scattering to $U_{3, \min }$ valley is not allowed. (b) Three-dimensional plot of the overlap parameter variation, with the final-state point again chosen at the $U_{3, \min }$ point. The spherical angles of each data point correspond to those of the initial-state wave vector, and the radial distance gives the resultant overlap square.

the considered directions in Fig. 4; the deviation is somewhat higher around the $\mathrm{CB}$ edge for the $\Gamma-A$ direction.

To see the effects of the symmetry (i.e., cell-periodic parts of the participating wave functions), we have repeated the same computation by setting the overlap parameter $\Delta_{m^{\prime}, m}$ to unity, the results being shown in Fig. 4 by the dashed lines. Note that around the vicinity of the CB minimum this approximation can be employed, however, remarkable deviation takes place as soon as the energy level reaches $U_{3, \min }$ valley, leading to overestimation of the scattering to this valley. To further illustrate this point, in Fig. 5(a) we compare the overlap parameter between the $U_{3, \min }$ point and any point along the $\Gamma-A$ and $\Gamma-M$ directions. The primary purpose of this figure is to show that the actual overlap with $U_{3, \min }$ point

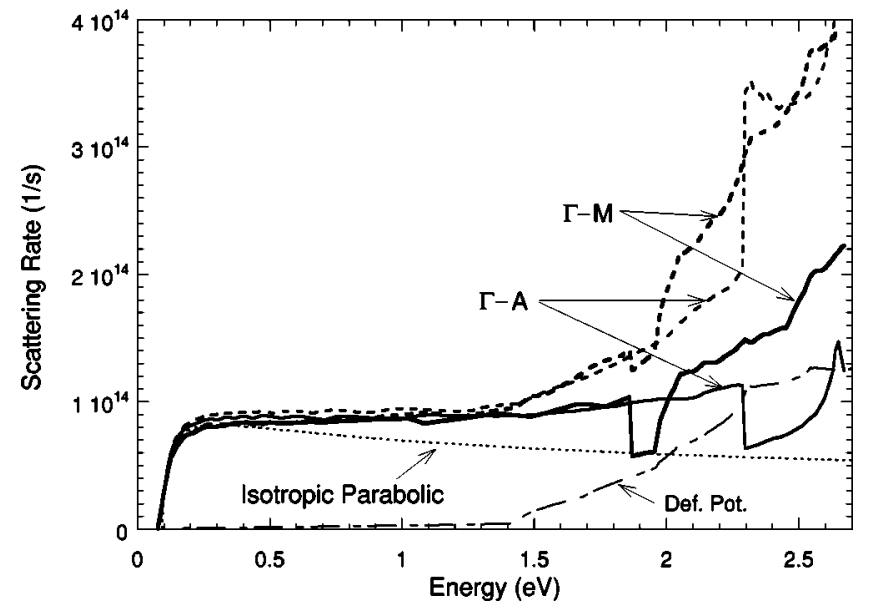

FIG. 6. Scattering rate versus energy, comparing the LO-like POP rates with the nonpolar deformation potential scattering; results apply up to room temperature. The energy reference is chosen to be at the CB minimum. The dashed lines correspond to the LOlike rates when the cell-periodic overlaps are taken to be unity. The dotted line shows the cubic isotropic parabolic POP rate.

is much less than unity. The interval between the dashed lines corresponds to energies where POP scattering to $U_{3, \text { min }}$ valley is not allowed. Still retaining the final electron state as the $U_{3, \min }$ point, we now scan over all initial electron states that can be scattered to $U_{3, \min }$ point via an LO-like POP emission process. Figure 5(b) shows the resultant overlap parameter variation over the spherical angles defining the initial state of the electron. It is seen that the overlap parameter and hence the scattering rate to $U_{3, \min }$ point from $\Gamma-M$ direction is about 1.5 times higher than that of the $\Gamma-A$ direction.

From an alternative perspective, Fig. 6 displays the scattering rate expressions versus energy using Eq. (13). When the energy of the $M_{1}$ satellite valley is reached at $1.87 \mathrm{eV}$, the average scattering rate suddenly drops as electrons at the bottom of this valley contribute very little to POP scattering rate, limited to intervalley scattering with large phonon wave vectors. However, when the energy is increased further to allow the electrons in $M_{1}$ valley to have intravalley scattering, the rate rapidly rises. Also note that the carriers in the $M_{1}$ valley do not undergo an intervalley scattering to the $U_{3, \min }$ valley due to symmetry reasons, as displayed on the far left part of Fig. 5(a). In Fig. 6, we also show the POP scattering rate based on the isotropic parabolic band model, which only agrees well within $\sim 0.4 \mathrm{eV}$ range of the $\mathrm{CB}$ minimum.

For comparison purposes, we further include an estimate for the nonpolar LO deformation potential scattering rate, given at zero-temperature by ${ }^{2} \quad W(E)=\pi D_{\mathrm{LO}}^{2} N(E$ $\left.-\hbar \omega_{\mathrm{LO}}\right) / \rho \omega_{\mathrm{LO}}$, where $\rho$ is the mass density (taken as 6.1 $\mathrm{g} / \mathrm{cm}^{3}$ ) and $D_{\mathrm{LO}}$ is the LO deformation potential field. $N()$ is the single-spin DOS per volume which can be obtained from that given in Fig. 2 by noting that the corresponding "volume'" of an atom is $a^{2} c \sqrt{3} / 8$. We also ignore the nonpolar scattering due to other phonon polarizations and branches and use an overall value for the deformation potential as $10^{9}$ $\mathrm{eV} / \mathrm{cm}$, which applies to GaAs intervalley transitions. The resultant nonpolar rate is seen to be well below the POP rate 
over a great portion of the energies of the lowest CB. However, a strict quantitative comparison is not very reliable, given the gross uncertainity in the deformation potential field values for $\mathrm{GaN}$ and the simplistic treatment used here for the deformation potential scattering.

\section{IMPLICATIONS ON BLOCH OSCILLATIONS AND NEGATIVE DIFFERENTIAL CONDUCTIVITY}

\section{A. Bloch oscillations}

Our investigation of the full-band directional scattering analysis in GaN leads to some implications about the Bloch oscillations and the negative differential conductivity. Though, in superlattices these Bloch oscillations were observed through optical experiments (for a review see, e.g., Ref. 30), it is yet to be seen in transport experiments ${ }^{31}$ and in bulk semiconductors. The existence of Bloch oscillations is possible in a window of electric fields, limited from below by the scattering mechanisms, breaking the phase memory of the participating electrons, and from above by the escape of the electron to other bands or the breakdown of the material by Zener or impact ionization mechanisms. Our work here can only address the lower limit set on the electric field by the dominant scattering mechanism, this being the LOphonon emission up to room temperatures. As for the breakdown voltage, wide bandgap semiconductors, such as III nitrides (such as $\mathrm{GaN}$ ) presumably offer higher breakdown values, tempting further research in this direction. ${ }^{3}$

Bloch oscillation corresponds to the traversal of an electron along a RLV from one reciprocal lattice point to another forming a repetitive motion. Strictly speaking this regime requires the applied electric field direction to coincide with a RLV. Under these circumstances, if the magnitude of the shortest RLV along the field direction is $|\mathbf{G}|$, then the Bloch angular frequency simply follows from the acceleration theorem as $\omega_{B}=2 \pi e F / \hbar|\mathbf{G}|$. For the case of the $\mathrm{WZ}$ crystal, the two shortest RLVs are along $\Gamma-M$ and $\Gamma-A$ directions with the magnitudes $4 \pi / a \sqrt{3}$ and $2 \pi / c$, respectively. However, for the $\Gamma-A$ direction, twice this value (hence $4 \pi / c$ ) needs to be considered due to BZ folding effect (see Fig. 3).

The criterion for the observation of Bloch oscillations shows some variation over the literature. ${ }^{19,30,32-36}$ A common expression $^{19,30,32}$ is $\omega_{B} \tau>1$, which is based on the fact that the associated Wannier-Stark level spacing $\hbar \omega_{B}$ being greater than the level broadening, $\hbar / \tau$ due to scattering, where $\tau$ is the (maximum) scattering time within the band. Instead, von Plessen et al. ${ }^{33}$ suggest $\hbar / \tau$ to be replaced by $\Gamma_{\text {hom }}=2 \hbar / \tau$, which is the homogeneous linewidth related to scattering time $\tau$. A rather more strict criterion put forward by Esaki and Tsu is $\omega_{B} \tau>2 \pi$, which requires the electron to complete one cycle in the BZ before undergoing a scattering event. ${ }^{34}$ If we adopt this last criterion by Esaki and Tsu, then the threshold field for the onset of the the Bloch oscillations turns out to be well above $10 \mathrm{MV} / \mathrm{cm}$, which cannot be attained due to the dielectric breakdown of the material.

\section{B. Negative differential conductivity}

A precursor of Bloch oscillations is the negative differential conductivity (NDC) at lower fields. There have already

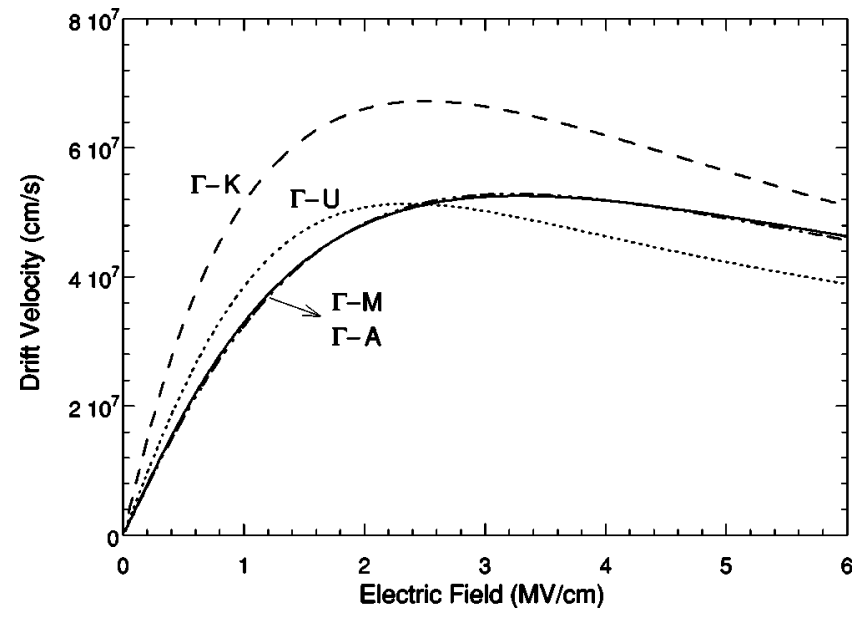

FIG. 7. High-field drift velocity dependence along four different directions. The $\Gamma-M$ and $\Gamma-A$ curves almost coincide.

been several predictions of NDC in GaN (Refs. 37$39,18,40)$ at field levels of around $150 \mathrm{kV} / \mathrm{cm}$, all being based on the transfer of electrons to upper valleys, which was also experimentally verified with reasonable agreement. ${ }^{41}$ On the other hand, the NDC that here we refer to, is driven by the band structure's negative effective mass part beyond the inflection point of its dispersion curve, an idea that goes back to Krömer. ${ }^{42}$ Esaki and Tsu have later discussed this mechanism of NDC for the case of superlattices. ${ }^{34}$ For this reason, it can be distinguished as the Krömer-Esaki-Tsu NDC. Recently, the possibility of this mechanism over the valley transfer one was advocated for the case of cubic GaN. ${ }^{43}$

The dependence of drift velocity on the applied electric field can easily be estimated by the simple approach used by Esaki and Tsu for superlattices. ${ }^{34}$ Hence, the average drift velocity assuming a constant scattering time $\tau$ is given by

$$
v_{d}=e F \hbar^{-2} \int_{0}^{\infty} \frac{\partial^{2} E_{F l d}}{\partial k_{F l d}^{2}} e^{-t / \tau} d t,
$$

where $\partial^{2} E_{F l d} / \partial k_{F l d}^{2}$ is the curvature of the energy band diagram along the applied field's direction $\mathbf{F}$, sampled at the $\mathbf{k}$ point, $k_{F l d}(t)=e F t / \hbar$, for an electron originating from the $\Gamma$ point. For the scattering time $\tau$ in this equation, we use the value given by the maximum scattering rate in Fig. 4 in each direction. Figure 7 shows the corresponding drift velocity dependence on the electric field. The different low-field mobilities are the artifact of using the maximum scattering rate of each direction throughout the band. Actually, this lowfield part of the drift velocity is governed by the momentum relaxation time, and of course, it is not described correctly by the Esaki-Tsu formula Eq. (14). However, the concept of momentum relaxation time refers to a distribution of particles and is not relevant in the present context of high-field transport which is to do with the probability of avoiding collision and hence with the bare scattering time. With this reservation, Esaki-Tsu formula results are plotted for all the fields and therefore, only the high-field regime, above $\sim 1$ $\mathrm{MV} / \mathrm{cm}$ part should be considered. It is seen that the NDC has a significant dependence on the chosen direction of transport and needs high electric fields, above $2.3 \mathrm{MV} / \mathrm{cm}$. A very 
recent experiment, ${ }^{44}$ measuring the steady-state drift velocity in $\mathrm{GaN}$ has gone up to an electric field of $350 \mathrm{kV} / \mathrm{cm}$. Essentially a constant drift velocity of $2 \times 10^{7} \mathrm{~cm} / \mathrm{s}$ for fields above $200 \mathrm{kV} / \mathrm{cm}$ has been extracted. Our preliminary estimations in Fig. 7 suggest a substantial increase in the drift velocity at field levels about a few MV/cm. This may have an important practical implication for GaN-based highpower devices, so further experiments at such high fields will be very valuable to investigate this possibility.

\section{SUMMARY}

In summary, we have obtained the band structure and the associated Bloch wave functions of $\mathrm{WZ} \mathrm{GaN} \mathrm{using} \mathrm{EPM}$ paying due attention to $\mathrm{CB}$ properties in light of recent experiments. The merits and flaws of this band structure can be assessed experimentally by verifying our resultant data characterizing the $\mathrm{CB}$ satellite valleys. Using this band structure we analyzed the full-band LO-like and TO-like POP scattering rates along several important directions in the BZ. We have demonstrated the key roles played by the satellite valleys and the symmetry of the participating wave functions. More elaborate high-field transport calculations and experiments for bulk GaN will be very useful in further exploring the NDC possibilities. Our rough estimation suggests the threshold field for NDC in WZ GaN to be about $2.3 \mathrm{MV} / \mathrm{cm}$.

\section{ACKNOWLEDGMENTS}

This work was supported by Grant Nos. ONR (N0001499-1-0014) and EPSRC (GR/L/56725).

\section{APPENDIX: COMPUTATION OF THE FULL-BAND SCATTERING RATE}

\section{By direct integration}

We compute the scattering rate given by Eq. (6) in two alternative ways which agree with each other within numerical accuracy. The first approach is to follow a direct integration of the rate expression. As the initial standard step, the summation $\left(\Sigma_{\mathbf{k}^{\prime}}\right)$ in this equation is converted to integration $\left[V /(2 \pi)^{3} \int d^{3} k^{\prime}\right]$, the integration domain being the first BZ which is hexagonal in WZ structures. We enclose this hexagonal BZ by a cylindrical box with radius $4 \pi / 3 a$ and height $2 \pi / c$, and work in cylindrical coordinates for all the wave vectors. We discard the regions of the cylinder that lies outside the hexagonal BZ by inserting a function $\Theta_{\mathrm{BZ}}$, in the integrand (see the expressions below). In the rest of this subsection, we normalize all energies to atomic Rydbergs, the component of wave vectors in the hexagonal plane to $2 \pi / a$ and the component along the $c$ axis (which we choose to be along the $z$ axis) to $2 \pi / c$. With these manipulations and after applying the delta function property together with the associated coupling coefficients [Eqs. (9), (10)], Eq. (6) becomes

$$
\begin{aligned}
& W_{m}^{ \pm} \text {LO-like }(\mathbf{k})=\frac{2 a_{B}}{c} \int_{0}^{2 \pi} d \phi^{\prime} \int_{-1 / 2}^{1 / 2} d k_{z}^{\prime} \sum_{i} \frac{k_{\perp i}^{\prime}}{q_{\perp i}^{2}+\left(\frac{a}{c}\right)^{2} q_{z i}^{2}}\left(n_{L, \mathbf{q}_{i}}+\frac{1}{2} \pm \frac{1}{2}\right) \frac{\Theta\left(\frac{2}{3}-k_{\perp i}^{\prime}\right)}{\left|\frac{\partial f_{L}}{\partial k_{\perp i}^{\prime}}\right|} \Theta_{\mathrm{BZ}}\left(k_{\perp i}^{\prime}, \phi^{\prime}\right) \frac{\Delta_{m^{\prime}, m}\left(\mathbf{k}_{i}^{\prime}, \mathbf{k}\right)}{\Omega_{L}\left(\theta_{\mathbf{q}_{i}}\right)} \\
& \times\left[\frac{\sin ^{2} \theta_{\mathbf{q}_{i}}}{\left(\frac{1}{\epsilon_{\perp}^{\infty}}-\frac{1}{\epsilon_{\perp}^{0}}\right) \omega_{\perp L}^{2}}+\frac{\cos ^{2} \theta_{\mathbf{q}_{i}}}{\left(\frac{1}{\epsilon_{z}^{\infty}}-\frac{1}{\epsilon_{z}^{0}}\right) \omega_{z L}^{2}}\right]^{-1} \\
& W_{m, \text { TO-like }}^{ \pm}(\mathbf{k})=\frac{2 a_{B}}{c} \int_{0}^{2 \pi} d \phi^{\prime} \int_{-1 / 2}^{1 / 2} d k_{z}^{\prime} \sum_{i} \frac{k_{\perp i}^{\prime}}{q_{\perp i}^{2}+\left(\frac{a}{c}\right)^{2} q_{z i}^{2}}\left(n_{T, \mathbf{q}_{i}}+\frac{1}{2} \pm \frac{1}{2}\right) \frac{\Theta\left(\frac{2}{3}-k_{\perp i}^{\prime}\right)}{\left|\frac{\partial f_{T}}{\partial k_{\perp i}^{\prime}}\right|} \Theta_{\mathrm{BZ}}\left(k_{\perp i}^{\prime}, \phi^{\prime}\right) \\
& \times \frac{\Delta_{m^{\prime}, m}\left(\mathbf{k}_{i}^{\prime}, \mathbf{k}\right)\left(\omega_{\perp}^{2}-\omega_{z}^{2}\right)^{2} \sin ^{2} \theta_{\mathbf{q}_{i}} \cos ^{2} \theta_{\mathbf{q}_{i}}}{\Omega_{T}\left(\theta_{\mathbf{q}_{i}}\right)\left[\left(\epsilon_{\perp}^{0}-\epsilon_{\perp}^{\infty}\right) \omega_{\perp}^{2} \cos ^{2} \theta_{\mathbf{q}_{i}}+\left(\epsilon_{z}^{0}-\boldsymbol{\epsilon}_{z}^{\infty}\right) \omega_{z}^{2} \sin ^{2} \theta_{\mathbf{q}_{i}}\right]},
\end{aligned}
$$

where $a_{B}$ is the atomic Bohr radius, $\mathbf{k}_{i}^{\prime}$ with the corresponding cylindrical components $\left(k_{\perp i}^{\prime}, \phi^{\prime}, k_{z}^{\prime}\right)$ is the $i$ th zero of the function $f_{L, T}=E_{m^{\prime}}\left(\mathbf{k}^{\prime}\right)-E_{m}(\mathbf{k}) \pm \hbar \Omega_{L, T}$ and $\quad \mathbf{q}_{i}=\mp\left(\mathbf{k}_{i}^{\prime}\right.$ -k) mapped to first BZ; $\Theta$ is the step (Heaviside) function and $\Theta_{\mathrm{BZ}}$ is the function that yields 1 (0) if $\mathbf{k}_{\perp i}^{\prime}$ lies inside (outside) the first hexagonal BZ. For the special cases of the initial-state electron wave vector $\mathbf{k}$, we have certain simplifications due to symmetry: if $k_{z} \equiv 0$, then $\int_{-1 / 2}^{1 / 2} d k_{z}^{\prime}$ $\rightarrow 2 \int_{0}^{1 / 2} d k_{z}^{\prime}$, and if $\mathbf{k} \equiv \hat{z} k_{z}$, then $\int_{0}^{2 \pi} d \phi^{\prime} \rightarrow 12 \int_{0}^{\pi / 6} d \phi^{\prime}$.

The computational bottleneck lies in finding the zeros of the function $f_{L, T}$. Here, we employ the linear tetrahedron interpolation of the band energies. ${ }^{45}$ For this purpose, we store the CB energies by sampling the irreducible BZ (IBZ) with 22050 data points, giving more emphasis to the vicinity of the $\Gamma$ point. The cell-periodic Bloch overlap function $\Delta_{m^{\prime}, m}$ is smoother (see Fig. 5), and a 910-point final-state 
sampling over the IBZ is used. Note that $\Delta_{m^{\prime}, m}$ does not require the phase information of the final state and only IBZ is sufficient for tabulating this function, whereas in Monte Carlo simulations the correct phase in other portions of the BZ needs to be generated to trace each particle. ${ }^{46,27}$ Finally, we include the lowest four $\mathrm{CB}$ for the summation over the final-state bands $m^{\prime}$ in Eq. (6). Actually only two bands are sufficient for all the considered directions but the $\Gamma-K$ direction, which goes up in energy after a band crossing (see Fig. $1)$.

\section{By the Lehmann-Taut technique}

The first approach utilizes the division of the IBZ into small tetrahedra simply for the interpolation of the energy and overlap parameters. Alternatively, using this infrastructure, the tetrahedron integration technique of Lehmann and Taut ${ }^{45}$ can be applied for the overall computation of the scattering rate. For this purpose, Eq. (6) can be put into the form

$$
W_{j, m}^{ \pm}(\mathbf{k})=\sum_{m^{\prime}} \int_{S} d S \frac{\hat{W}_{j, m^{\prime}}^{ \pm}\left(\mathbf{k}^{\prime}\right)}{\left|\nabla_{\mathbf{k}^{\prime}} E_{m^{\prime}} \mathbf{k}^{\prime}\right|},
$$

where

$$
\hat{W}_{j, m^{\prime}}^{ \pm}\left(\mathbf{k}^{\prime}\right)=\frac{2 \pi}{\hbar} \frac{V}{(2 \pi)^{3}} \Delta_{m^{\prime}, m}\left(\mathbf{k}^{\prime}, \mathbf{k}\right)\left|C_{j}(\mathbf{q})\right|^{2}\left(n_{j, \mathbf{q}}+\frac{1}{2} \pm \frac{1}{2}\right) .
$$

The integration is over the surface $S$ described by the energy conservation relation of one-phonon-emission-absorption process, $E_{m^{\prime}}\left(\mathbf{k}^{\prime}\right)=E_{m}(\mathbf{k}) \mp \hbar \omega_{j, \mathbf{q}}$. Equation (A3) is in the form for which Lehmann-Taut algorithm ${ }^{45}$ is directly applicable. Accordingly, the IBZ is divided into fine tetrahedra; the grid sizes for the storage of energy and overlap parameters are given in the previous subsection. However, the quantity $\hat{W}_{j, m^{\prime}}^{ \pm}\left(\mathbf{k}^{\prime}\right)$ needs much finer tetrahedra (about half a million) but need not be stored in the memory as it can be very easily calculated in real time. We have observed the Lehmann-Taut technique to be much more robust and computationally efficient than the previously explained direct integration approach.
${ }^{1}$ S. Nakamura and G. Fasol, The Blue Laser Diode (Springer, Berlin, 1997).

${ }^{2}$ B. K. Ridley, in Gallium Nitride and Related Materials II, edited by C. R. Abernathy, H. Amano, and J. C. Zolper, MRS Symposia Proceedings No. 468 (Materials Research Society, Pittsburgh, 1997), p. 231.

${ }^{3}$ B. K. Ridley, J. Phys.: Condens. Matter 10, L607 (1998).

${ }^{4}$ A. García and M. L. Cohen, Phys. Rev. B 47, 4215 (1993); 47, 4221 (1993).

${ }^{5}$ M. L. Cohen and V. Heine, Solid State Physics: Advances in Research and Applications, edited by H. Ehrenreich, F. Seitz, and D. Turnbull (Academic, New York, 1970), Vol. 24.

${ }^{6}$ S. Bloom, J. Phys. Chem. Solids 32, 2027 (1971).

${ }^{7}$ R. Wang, P. P. Ruden, J. Kolnik, I. Oguzman, and K. F. Brennan, J. Phys. Chem. Solids 58, 913 (1997).

${ }^{8}$ Y. C. Yeo, T. C. Chong, and M. F. Li, J. Appl. Phys. 83, 1429 (1998).

${ }^{9}$ S. K. Pugh, D. J. Dugdale, S. Brand, and R. A. Abram, J. Appl. Phys. 86, 3768 (1999).

${ }^{10}$ J. B. Xia, K. W. Cheah, X. L. Wang, D. Z. Sun, and M. Y. Kong, Phys. Rev. B 59, 10119 (1999).

${ }^{11}$ T. Valla, P. D. Johnson, S. S. Dhesi, K. E. Smith, D. Doppalapudi, T. D. Moustakas, and E. L. Shirley, Phys. Rev. B 59, 5003 (1999).

${ }^{12}$ C.-K. Sun, Y.-L. Huang, S. Keller, U. K. Mishra, and S. P. DenBaars, Phys. Rev. B 59, 13535 (1999).

${ }^{13}$ R. Loudon, Adv. Phys. 13, 423 (1964).

${ }^{14}$ M. Drechsler, D. M. Hofmann, B. K. Meyer, T. Detchprohm, H. Amano, and I. Akasaki, Jpn. J. Appl. Phys. 34, L1178 (1995).

${ }^{15}$ V. J. Bellitto, B. D. Thoms, D. D. Koleske, A. E. Wickenden, and R. L. Henry, Phys. Rev. B 60, 4816 (1999).

${ }^{16}$ J. W. Orton and C. T. Foxon, Rep. Prog. Phys. 61, 1 (1998).

${ }^{17}$ See, e.g., W. H. Press, S. A. Teukolsky, W. T. Vetterling, and B. P. Flannery, Numerical Recipes in FORTRAN, 2nd ed. (Cambridge University Press, Cambridge, 1992), p. 436.
${ }^{18}$ J. Kolník, İ. H. Oğuzman, K. F. Brennan, R. Wang, P. P. Ruden, and Y. Wang, J. Appl. Phys. 78, 1033 (1995).

${ }^{19}$ B. K. Ridley, Quantum Processes in Semiconductors, 4th ed. (Oxford University Press, Oxford, 1999).

${ }^{20}$ H. Fröhlich, Adv. Phys. 3, 325 (1954).

${ }^{21}$ B. C. Lee, K. W. Kim, M. Dutta, and M. A. Stroscio, Phys. Rev. B 56, 997 (1997).

${ }^{22}$ For the lowest $\mathrm{CB}$ this number is unity below the energy of the satellite valley in that direction, and becomes two above the energy of the valley (see Fig. 1).

${ }^{23}$ J. Bardeen and W. Shockley, Phys. Rev. 80, 69 (1950).

${ }^{24}$ Bardeen-Shockley analysis (Ref. 23) is based on the so-called Houston wave function (Ref. 25) that takes into account the electric field. It should be noted that for very high fields the Houston wave function treatment becomes less reliable and intracollisonal field effects come into play for fields on the order of MV/cm (Ref. 19).

${ }^{25}$ W. V. Houston, Phys. Rev. 57, 184 (1940).

${ }^{26} U$ symmetry line connects the points $M$ and $L$, and attains its $\mathrm{CB}$ minimum $\left(U_{3, \min }\right)$ on this line two thirds away from the $M$ points (see the inset to Fig. 1).

${ }^{27}$ I. H. Oğuzman, J. Kolník, K. F. Brennan, R. Wang, T. N. Fang, and P. P. Ruden, J. Appl. Phys. 80, 4429 (1996).

${ }^{28}$ T. Azuhata, T. Sota, K. Suzuki, and S. Nakamura, J. Phys.: Condens. Matter 7, L129 (1995).

${ }^{29}$ This creates a superstructure along the $c$ axis. It is worth mentioning that another wide bandgap material $\mathrm{SiC}$, has a stable polytype $6 \mathrm{H} \mathrm{SiC}$, having six hexagonally closed packed layers along the $c$ axis.

${ }^{30}$ P. Voisin, Ann. Phys. (N.Y.) 22, 681 (1997).

${ }^{31}$ A. Di Carlo, P. Vogl, and W. Pötz, Phys. Rev. B 50, 8358 (1994).

${ }^{32}$ E. E. Mendez and G. Bastard, Phys. Today 46, 34 (1993).

${ }^{33}$ G. von Plessen, T. Meier, J. Feldmann, E. O. Göbel, P. Thomas, K. W. Goossen, J. M. Kuo, and R. F. Kopf, Phys. Rev. B 49, 14058 (1994). 
${ }^{34}$ L. Esaki and R. Tsu, IBM J. Res. Dev. 14, 61 (1970).

${ }^{35}$ R. O. Grondin, W. Porod, J. Ho, D. K. Ferry, and G. J. Iafrate, Superlattices Microstruct. 1, 183 (1985).

${ }^{36}$ F. Beltram, F. Capasso, D. L. Sivco, A. L. Hutchinson, S. G. Chu, and A. Y. Cho, Phys. Rev. Lett. 64, 3167 (1990).

${ }^{37}$ M. A. Littlejohn, J. R. Hauser, and T. H. Glisson, Appl. Phys. Lett. 26, 625 (1975).

${ }^{38}$ B. Gelmont, K. Kim, and M. Shur, J. Appl. Phys. 74, 1818 (1993).

${ }^{39}$ N. S. Mansour, K. W. Kim, and M. A. Littlejohn, J. Appl. Phys. 77, 2834 (1995).

${ }^{40}$ B. E. Foutz, S. K. O'Leary, M. S. Shur, and L. F. Eastman, J.
Appl. Phys. 85, 7727 (1999).

${ }^{41}$ Z. C. Huang, R. Goldberg, J. C. Chen, Y. Zheng, D. B. Mott, and P. Shu, Appl. Phys. Lett. 67, 2825 (1995).

${ }^{42}$ H. Krömer, Phys. Rev. 109, 1856 (1958).

${ }^{43}$ S. Krishnamurthy, M. van Schilfgaarde, A. Sher, and A.-B. Chen, Appl. Phys. Lett. 71, 1999 (1997).

${ }^{44}$ M. Wraback, H. Shen, J. C. Carrano, T. Li, J. C. Campbell, M. J. Schurman, and I. T. Ferguson, Appl. Phys. Lett. 76, 1155 (2000).

${ }^{45}$ G. Lehmann and M. Taut, Phys. Status Solidi B 54, 469 (1972).

${ }^{46}$ N. Sano and A. Yoshii, Phys. Rev. B 45, 4171 (1992). 